\title{
The sterility and sterilization of lung ventilators
}

\author{
F. J . B AKER, B. G. B. LUCAS, A N D A. B. SEIBER \\ From the Brompton Hospital, London
}

Intermittent positive pressure breathing machines are in use for the treatment of many respiratory and cardiac conditions, but there is no agreement regarding the risk of cross infection and the necessity of sterilizing part, or all, of this equipment. Until recently, complete sterilization has been difficult to effect, and so reliance has been placed on boiling or chemical disinfection of the breathing tubing only. During an outbreak of unexplained post-operative respiratory complications in one respiratory unit amongst patients who had been ventilated on positive pressure breathing machines, the same organisms were found to be common to both the patients and the machines. We therefore investigated this to see whether, if machines become infected by patients, prevention is possible.

For the experiments sterile Radcliffe positive negative ventilators were used. Complete sterilization can be achieved by exposing a machine to a mixture of $10 \%$ ethylene oxide gas in an atmosphere of carbon dioxide over a period of time, provided that the machine is run continuously during the process so that the gas permeates all parts (Baker and Seaber, 1962). The complete machine, with the breathing tubes detached and the humidifier empty, is placed in a large plastic bag, which is made gas-tight with special zip fasteners, and the electric cable is led to a suitable power point through a sleeve in the bag. Air is exhausted from the bag as far as possible by applying suction from a domestic vacuum cleaner. The bag is filled with the mixture of ethylene oxide and carbon dioxide and sealed, and the machine is run in this atmosphere for 12 hours. The apparatus is then removed and, with the machine still running, flushed through with air for two hours. The efficacy of this method has been checked by deliberately infecting machines with a 24-hr. broth culture of Staphylococcus aureus of known phage type, which was sprayed on to the inner surfaces of all components of the ventilators. After sterilization, swabs were taken from all the infected parts, plated on to a blood agar plate, and inoculated into tubes of nutrient broth. On no occasion was any growth detected, although controls set up at the same time and not exposed to ethylene oxide all showed growth. Flushing with air after sterilization was necessary in order to remove all traces of ethylene oxide. This gas is absorbed on to the surface of rubber and some plastic materials and might be given off into the breathing circuit later. The time of two hours (Fig. 1) was determined by frequent sampling of the effluent and estimating the ethylene oxide by a standard gas detector method (H.M.S.O., 1951).

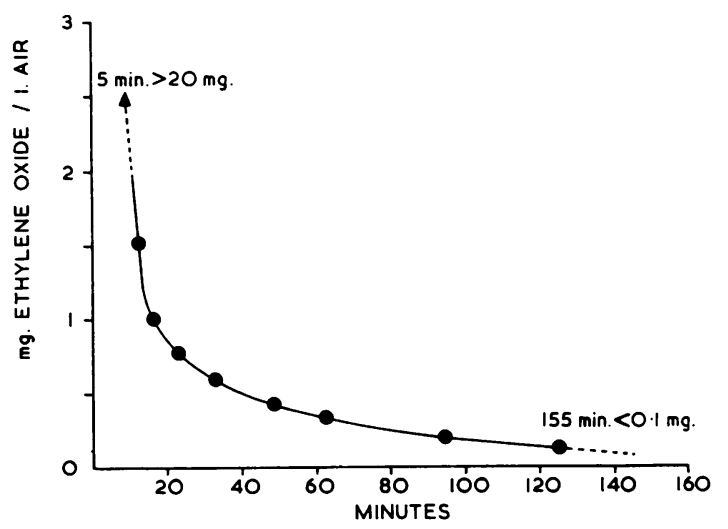

FIG. 1. Elimination of ethylene oxide from ventilator.

It was now possible to find out which parts of the ventilators become infected in normal use. A study of the pumping circuit (Fig. 2) shows that the only way in which the inlet side could be contaminated would be via the inlet port, that is by infected air being drawn into the machine, so one would only expect contamination as far as the bellows and humidifier, not beyond, as the humidifier should act as a partial bacteriological filter. Contamination would be greatest at the inlet port and least at the tracheotomy mount, but this has not been found to be so in practice. Cultures taken from machines that had been used on patients have shown that the heaviest infection on the in- 


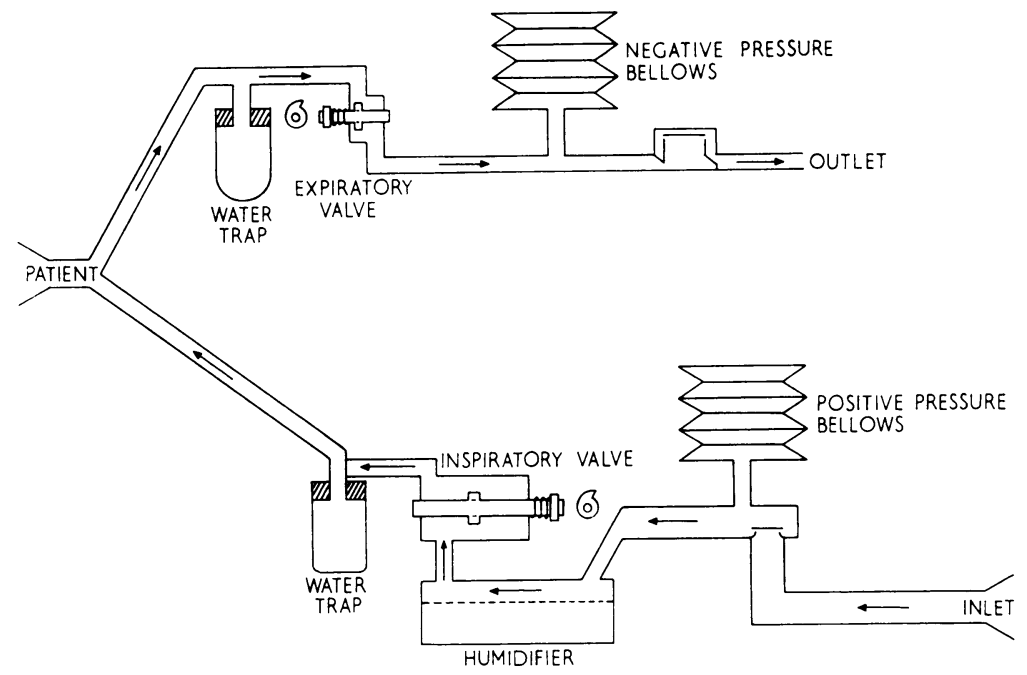

FIG. 2. Schematic diagram of Radcliffe positive negative ventilator.

spiratory side is between the inspiratory valve and the humidifier. This suggests that either the valves in the circuit are faulty, so allowing infected air to be blown back in the inspiratory circuit, or that pressure or flow changes in the inspiratory tubing are allowing infection to build up during the passage of air after it has passed through the humidifier.

Two experiments were devised to find out how ventilators become infected. The first was to determine the extent to which infection could be due to airborne organisms. A semi-rigid P.V.C. bag of about 100 litres capacity was sealed on to the inlet port of a newly sterilized ventilator. This bag had an inlet pipe open to the air, which was partially plugged with wool to act as a bacteriological filter. The machine was run continuously for 24 hours, and an aerosol spray of a 24-hr. broth culture of Staph. aureus was injected into the bag from time to time during this period. On dismantling the machine heavy contamination was found from the inlet port to the bellows and as far as the humidifier, but there was very little in the inspiratory tubing, showing that the water in the humidifier does act as a bacteriological filter. In the second experiment an infected artificial lung, composed of a rubber bag of 5 litres capacity partially filled with a 24-hr. broth culture of Staph. aureus, was connected to a sterile breathing machine. The machine was then run for 24 hours so that the bag continually inflated and deflated. On dismantling, it was found that the inspiratory tubing was contaminated as far back as the humidifier. This showed that organisms were being carried back along the inspiratory tube and past the inspiratory valve during some phase of the respiratory cycle.

The mechanism whereby bacteria are carried back beyond the inspiratory valve is interesting. Montgomery (1962, personal communication) has shown that in a simple gas system when a valve shuts there is a temporary build-up of pressure in the tube immediately behind the valve, which reverses the flow of gas around the periphery on either side of the valve. This back-flow on the upstream side will mix with that which has leaked past the valve during the moment of closure and move it further upstream. In lung ventilators this leakage will be contaminated, and bacteria will be deposited on the side of the tubing adjoining the valve. With the next positive stroke the bacteria will be carried a little further. This process has been demonstrated by repeating the second experiment and examining the inspiratory side for contamination after running the breathing machine for different periods of time. After one hour only the area nearest the artificial lung was found to be contaminated, and after 24 hours the whole inspiratory side as far as the humidifier.

These experiments show that cross infection can occur in intermittent positive pressure breathing machines, and that filtering the air inspired into the machine, or obtaining fresh air from outside the building, will not prevent contamination. The main infection occurs via the patient's inspiratory tubing, due to a leakage of bacteria back past the inspiratory valve. The introduction of a bacteriological filter in the inspiratory tubing might prevent this, but it would be unacceptable from a safety point of view as complete blockage of the filter could occur under certain circum- 
stances rendering artificial ventilation impossible. The only satisfactory solution to the problem is to sterilize the whole machine completely after use. The technique described, using ethylene oxide, has been found to be satisfactory.

\section{REFERENCES}

Baker, F. J., and Seaber, A. (1962). Progress in Medical Laboratory Technique I, p. 109. Butterworth, London.

H.M.S.O. (1951). Fumigation with Ethylene Oxide-Precautionary Measures. Appendix. 